\title{
Hypertrophic Cardiomyopathy: The Time-Synchronized Relationship between Ischemia and Left Ventricular Dysfunction Assessed by Highly Sensitive Troponin I and NT-proBNP
}

\author{
Renata Rajtar-Salwa, ${ }^{1}$ Adam Gębka, ${ }^{1}$ Artur Dziewierz, ${ }^{1,2}$ and Pawel Petkow Dimitrow $\mathbb{D}^{1,2}$ \\ ${ }^{1}$ Second Department of Cardiology and Cardiovascular Interventions, University Hospital, Krakow, Poland \\ ${ }^{2}$ Second Department of Cardiology, Jagiellonian University Collegium Medicum, Krakow, Poland \\ Correspondence should be addressed to Paweł Petkow Dimitrow; dimitrow@mp.pl
}

Received 22 January 2019; Revised 22 March 2019; Accepted 15 April 2019; Published 20 June 2019

Guest Editor: Zhongjie Shi

Copyright (C) 2019 Renata Rajtar-Salwa et al. This is an open access article distributed under the Creative Commons Attribution License, which permits unrestricted use, distribution, and reproduction in any medium, provided the original work is properly cited.

\begin{abstract}
The aim of this study was to compare NT-proBNP using the absolute values and NT-proBNP/ULN values that were standardized by age and gender between three subgroups: those without ischemia (negative hs-troponin I and no anginal pain (hsTnI-/AP-)), those with painless ischemia (hsTnI+/AP-), and those with painful ischemia (hsTnI+/AP+). Additionally, echocardiographic parameters were compared in these three subgroups. The absolute value of NT-proBNP was significantly higher in the painful ischemia subgroup (hsTnI-/AP- vs. hsTnI+/AP- vs. hsTnI+/AP+: 502 (174-833) vs. 969 (363-1346) vs. 2053 (323-3283) pg/ml; $p=0.018$ for the whole-model analysis). The standardized value of NT-proBNP/ULN was gradually increased (hsTnI-/AP- vs. hsTnI+/AP- vs. hsTnI+/AP+: $3.61+0.63$ vs. $6.90+1.31$ vs. $9.35+1.87 ; p=0.001$ for the whole-model analysis). In the comparison between subgroups (hsTnI-/AP- vs. hsTnI+/AP- vs. hsTnI+/AP+), two echocardiographic parameters increased significantly. The left ventricular maximum wall thickness (LVMWT) at diastole was $1.99 \pm 0.08 \mathrm{~cm}$ vs. $2.28 \pm 0.13 \mathrm{~cm}$ vs. $2.49 \pm 0.15 \mathrm{~cm}$ ( $p=0.004$ for the whole-model analysis). The maximal gradient of the provoked left ventricular outflow tract (LVOT) gradient increased significantly in only the painful-ischemia subgroup (11 (7-30) $\mathrm{mmHg}$ vs. 12 (9.35-31.5) $\mathrm{mmHg}$ vs. 100 (43-120) $\mathrm{mmHg}$ ). In conclusion, both painless ischemia and painful ischemia are associated with a gradual, significant increase in NT-proBNP/ULN in comparison to the double-negative hsTnI/AP subgroup. In contrast, NT-proBNP is significantly higher in only the subgroup with painful ischemia.
\end{abstract}

\section{Introduction}

It is proposed $[1,2]$ that routine measurement biomarkers especially including [1] both N-terminal pro-B-type NTpronatriuretic peptide (NT-proBNP) and cardiac troponin (Tn) may be useful in the clinical evaluation and management of patients with HCM. NT-proBNP is predominantly secreted from the ventricles in response to increased myocyte stretching from increased stress and pressure at the LV wall. It is plausible that microvascular ischemia directly stimulates the release of NT-proBNP in HCM. In clinical studies, there is little information about the combined use of Tn and plasma BNP as prognostic biomarkers for adverse events mediated by myocardial ischemia with LV dysfunction [3].
In a study by Kubo et al. [3] on 167 patients with HCM, $\mathrm{TnI}$ and BNP were measured only once at the initial examination without control measurements during a follow-up period of more than 3 years (mean value). Patients with elevated $\mathrm{TnI}$ values had more frequent adverse events. Similarly, the risk of adverse events was higher in patients with high BNP ( $\geq 200 \mathrm{pg} / \mathrm{ml})$. Importantly, TnI used in combination with BNP further improved the prognostic value, as patients with high values of both $\mathrm{cTnI}$ and BNP had nearly 12 times higher risk of cardiovascular events than patients with a combination of low cTnI/BNP values. However, this study had some important limitations because there was a long time between the initial biomarker measurement and the final event. 
In contrast, we studied the direct short-term relationship between angina pectoris and the levels of both hsTnI and NT-proBNP after a 24-hour monitoring period. Taking into account methodological aspects, we compared NT-proBNP using absolute values and NT-proBNP/ULN values that were standardized by age and gender between three subgroups: those without ischemia (negative hs-troponin I/no anginal pain (hsTnI-/AP-)), those with painless ischemia (hsTnI +/AP-), and those with painful ischemia (hsTnI+/AP+).

\section{Methods}

A total of 64 patients with HCM were recruited (mean age $37 \pm 6$ years, 33 men and 31 women). The study protocol was approved by the local institutional review board (Komisja Bioetyki Jagiellonian University KBET/119/B/2017). Informed consent was obtained from each participant. All patients met the standard diagnostic criteria for HCM [4]. In adults, HCM is defined by a wall thickness $\geq 15 \mathrm{~mm}$ in one or more LV myocardial segments that is not explained solely by loading conditions and measured by any imaging technique (echocardiography (our method), cardiac magnetic resonance imaging (CMR), or computed tomography (CT)) [4]. Patients on current pharmacotherapy or without pharmacotherapy (newly diagnosed patients referred to our ambulatory clinic) were examined by echocardiography with LVOT gradient provocation by a combination of two natural stimuli (orthostatic test and the Valsalva test).

The exclusion criteria were myocardial infarction with ST-segment or non-ST-segment elevation (current or previous), previous alcohol septal ablation, significant coronary stenosis in recent coronary angiography, diabetes mellitus, regular sports activity, dilated LV cavity and decreased LV contractibility (we included only patients with LVEF $>50 \%$ ), atrial fibrillation, and elevated serum creatinine levels resulting in eGFR $<60 \mathrm{ml} / \mathrm{min}$. We included only patients with coronary microvessel disease, which is a common abnormality in HCM at any age (inclusion criteria: normal/near-normal coronary arteries or no indication of coronary arteriography).

The minority of patients did not have coronary angiography performed (young, without AP, without risk factors for CAD-especially without diabetes mellitus-see below). The risk for CAD was minimal and coronary angiography was not indicated. Diabetes mellitus is usually linked with silent ischemia from epicardial coronary arteries (so it is necessary to exclude painless macrovascular stenosis).

Renal failure is a typical extracardiac factor related to TnI elevation. Frequent sports activity may be responsible for repetitive myocardial ischemia in some patients [5]. Patients were asked to report presence or absence of angina pectoris episodes before the 24-hour period. Next, an echocardiographic examination was performed. Just after, hsTnI and NT-proBNP (absolute or upper limit of normal (ULN)) were measured. There were 38 patients in subgroup 0 (i.e., the double-negative group; hsTnI-/AP-). Subgroup 1 (hsTnI+/AP-) was composed of 12 patients, and subgroup 2 (double positive) consisted of 14 patients. A cut-off value of $19 \mathrm{ng} / \mathrm{l}$ was used according to the manufac-
TABLE 1: TNHS test repeatability and intralaboratory precision.

\begin{tabular}{lcc}
\hline $\begin{array}{l}\text { High-sensitivity troponin } \\
\text { (pg/ml; ng/l) }\end{array}$ & \multicolumn{2}{c}{ Assay output requirements } \\
$\begin{array}{c}\text { Repeatability } \\
\text { (within the same } \\
\text { series) }(\mathrm{CV}(\%))\end{array}$ & $\begin{array}{c}\text { Intralaboratory } \\
\text { precision (total) } \\
\text { (CV (\%)) }\end{array}$ \\
\hline $9-20$ & $\leq 10 \%$ & $\leq 12 \%$ \\
$>20$ & $\leq 10 \%$ & $\leq 10 \%$ \\
\hline
\end{tabular}

Precision was assessed in accordance with the EP05-A3 protocol of the Clinical and Laboratory Standards Institute (CLSI): "Evaluation of Precision of Quantitative Measurement Procedures; Approved Guidelines-Third Edition" (CLSI Document EP05-A3).

TABLE 2: NT-proBNP test repeatability and intralaboratory precision.

\begin{tabular}{|c|c|c|c|}
\hline \multicolumn{2}{|c|}{ NT-proBNP } & \multicolumn{2}{|c|}{ Assay output requirements } \\
\hline $\mathrm{pg} / \mathrm{ml}$ & $\mathrm{pmol} / \mathrm{l}$ & $\begin{array}{l}\text { Repeatability } \\
\text { (within the same } \\
\text { series) }(\mathrm{CV}(\%))\end{array}$ & $\begin{array}{l}\text { Intralaboratory } \\
\text { precision (total) } \\
(\mathrm{CV}(\%))\end{array}$ \\
\hline $100-500$ & $11.8-59.0$ & $\leq 5 \%$ & $\leq 8 \%$ \\
\hline$>500$ & $>59,0$ & $\leq 7 \%$ & $\leq 10 \%$ \\
\hline
\end{tabular}

Precision was assessed in accordance with the EP05-A3 protocol of the Clinical and Laboratory Standards Institute (CLSI): "Evaluation of Precision of Quantitative Measurement Procedures; Approved Guidelines-Third Edition" (CLSI Document EP05-A3).

turer's instructions (bioMerieux VIDAS ${ }^{\circledR}$ High sensitive Troponin I). This value represents the 99th percentile of a presumably healthy population.

High-sensitivity troponin tests were performed with the use of the VIDAS High sensitive Troponin I (TNHS). The test is capable of measuring cardiac troponin I concentration in the range of $4.9-40,000.00 \mathrm{pg} / \mathrm{ml}(\mathrm{ng} / 1)$ without the need for dilution. The TNHS test was designed to meet the following criteria of repeatability and intralaboratory precision (Table 1).

The NT-proBNP tests were performed with use of an Elecsys proBNP II Cobas e601 system. The test is capable of measuring the NT-proBNP concentration in the range of 5-35,000 $\mathrm{pg} / \mathrm{ml}$ without the need for dilution.

The proBNP II test was designed to meet the following criteria of repeatability and intralaboratory precision (Table 2).

The NT-proBNP levels were presented as absolute values and transformed values that were standardized according to sex and age based on the manufacturer's guidelines. (http:// www.rochecanada.com/content/dam/roche_canada/en_CA/ documents/package_inserts/ProBNPII-04842464190-EN-V9CAN.pdf). Values NT-proBNP greater than the 95th percentile for age and gender (the ULN) were considered abnormal.

Therefore, the results were expressed as the ratio of the NT-proBNP to age and sex-matched ULN. Ratios $>1.0$ were considered abnormal [6]. This standardization of NTproBNP provides a normal distribution of data, whereas absolute values were distributed abnormally. In this situation, we do not need to perform a logarithmic transformation for artificial calculation.

For the statistical analysis, continuous variables were presented as the mean ( \pm standard deviation $(\mathrm{SD})$ ) or median (interquartile range (IQR)). NT-proBNP levels were 
TABLE 3: The baseline characteristics of HCM patients.

(a)

\begin{tabular}{lr}
\hline Mean age & $37 \pm 6$ years \\
\hline Males/females & $33 / 31$ \\
The ICD implantation & 8 patients \\
EF \% & $59 \pm 8 \%$ \\
hsTnI value & $73.74 \pm 232.9$ \\
Medications & Patients \\
Beta-blockers & 43 \\
Verapamil & 17 \\
Diuretics & 3 \\
ACE inhibitors & 4 \\
Past history of AP in years & $4.1+1.2$ years \\
\hline
\end{tabular}

(b)

\begin{tabular}{|c|c|c|c|c|c|}
\hline \multicolumn{3}{|c|}{$\begin{array}{ll}\text { Total }(N=64) \\
\end{array}$} & Subgroup $0(N=38)$ & Subgroup $1(N=12)$ & Subgroup $2(N=14)$ \\
\hline \multicolumn{6}{|c|}{ Baseline characteristics of subgroups of patients with HCM } \\
\hline \multirow{3}{*}{ NYHA } & Class I ( $(\%))$ & $12(19 \%)$ & $9(24 \%)$ & $3(25 \%)$ & $0^{* * * *}$ \\
\hline & Class II $(n(\%))$ & $33(51 \%)$ & $20(52 \%)$ & $6(50 \%)$ & $7(50 \%)$ \\
\hline & Class III $(n(\%))$ & $19(30 \%)$ & $9(24 \%)$ & $3(25 \%)$ & $7(50 \%) *$ \\
\hline \multirow{3}{*}{ CCS } & Class I $(n(\%))$ & $24(38 \%)$ & $18(47 \%)$ & $5(42 \%)$ & $1(7 \%)^{* * * *}$ \\
\hline & Class II $(n(\%))$ & $29(45 \%)$ & $16(42 \%)$ & $5(42 \%)$ & $8(57 \%)$ \\
\hline & Class III $(n(\%))$ & $11(17 \%)$ & $4(11 \%)$ & $2(8 \%)$ & $5(36 \%)^{*}$ \\
\hline \multicolumn{2}{|c|}{ Syncope $(n(\%))$} & $25(39 \%)$ & $13(34 \%)$ & $5(42 \%)$ & $7(50 \%)$ \\
\hline \multicolumn{2}{|c|}{$\begin{array}{l}\text { Sudden death in family history } \\
(n(\%))\end{array}$} & $23(36 \%)$ & $14(37 \%)$ & $4(33 \%)$ & $5(36 \%)$ \\
\hline \multicolumn{6}{|c|}{ All patients had Holter } \\
\hline \multicolumn{2}{|c|}{ NSVT in Holter $(n(\%))$} & $26(41 \%)$ & $15(39 \%)$ & $5(42 \%)$ & $6(43 \%)$ \\
\hline \multicolumn{2}{|c|}{$\begin{array}{l}\text { LV maximal wall thickness } \\
(\mathrm{LVMWT}) \text { at diastole }(\mathrm{cm})\end{array}$} & $2.23 \pm 0.57$ & & Detailed calculation in Figure 3 & \\
\hline \multicolumn{2}{|c|}{$\begin{array}{l}\text { Resting LVOT gradient, } \geq 30 \\
\mathrm{mmHg}(n(\%))\end{array}$} & $14(22 \%)$ & $6(16 \%)$ & $3(25 \%)$ & $5(36 \%)$ \\
\hline \multicolumn{2}{|c|}{$\begin{array}{l}\text { Provocable LVOT gradient, } \\
\geq 30 \mathrm{mmHg}(n(\%))\end{array}$} & $14(22 \%)$ & $5(13 \%)$ & $3(25 \%)$ & $6(43 \%)^{*}$ \\
\hline \multicolumn{2}{|c|}{ Left atrial diameter $(\mathrm{cm})$} & $4.78 \pm 0.64$ & & Detailed calculation in Figure 5 & \\
\hline
\end{tabular}

Abbreviations: CCS: Canadian Cardiovascular Society; LVOT: left ventricular outflow tract; LV: left ventricular; NSVT: nonsustained ventricular tachycardia; NYHA: New York Heart Association. ${ }^{*} p<0.05$ subgroup 0 vs. $2 ;{ }^{* *} p<0.05$ subgroup 1 vs. 2.

compared between the three subgroups of patients using the Kruskal-Wallis test, which was also used to compare the maximal LVOT gradient. The values of standardized NTproBNP/ULN had a normal distribution according to the Kolmogorov-Smirnov test and were compared using ANOVA for comparison, which was also used to compare the LV maximum wall thickness (LVMWT) and left atrial diameter (LAD). Stepwise multiple linear regression analysis was used to identify factors independently correlated with NT-proBNP levels. Patients' age, gender, troponin level, presence of angina, and several echocardiographic parameters (max LVH, resting LVOT gradient, maximal LVOT gradient, and LAD) were tested as possible candidates. A $p$ value of $<0.05$ was considered statistically significant (Statistica 12.0).

\section{Results}

Demographics, relevant echocardiographic information, medical history, and treatment data are presented in Table 3.

Among all patients, chest pain was present more than 10 hours (during daily physical activity on the first day) before the blood sampling at 8.00 a.m. on the second day in the morning. There were no chest pains during the night.

The absolute value of NT-proBNP was significantly higher in the painful ischemia subgroup (hsTnI-/AP- vs. hsTnI+/AP- vs. hsTnI+/AP+: 502 (174-833) vs. 969 (363$1346)$ vs. 2053 (323-3283) pg/ml; $p=0.0178$ for the whole model, Figure 1). 


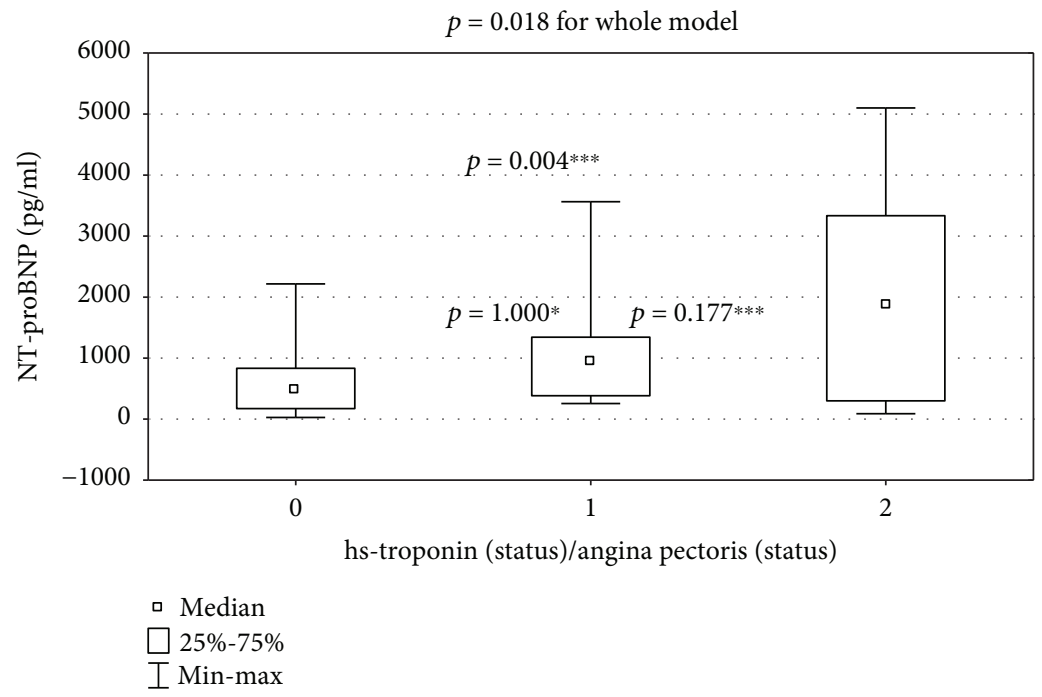

FIgURE 1: Comparison of NT-proBNP between subgroup 0 (hsTnI-/AP-), subgroup 1 (hsTnI+/AP-), and subgroup 2 (hsTnI+/AP+); $p=0.018$ for the whole model. For inter-subgroup comparison: * subgroup 0 vs. $1: p=1.000 ;{ }^{* *}$ subgroup 1 vs. $2: p=0.177 ;{ }^{* * *}$ subgroup 0 vs. $2: p=0.004$.

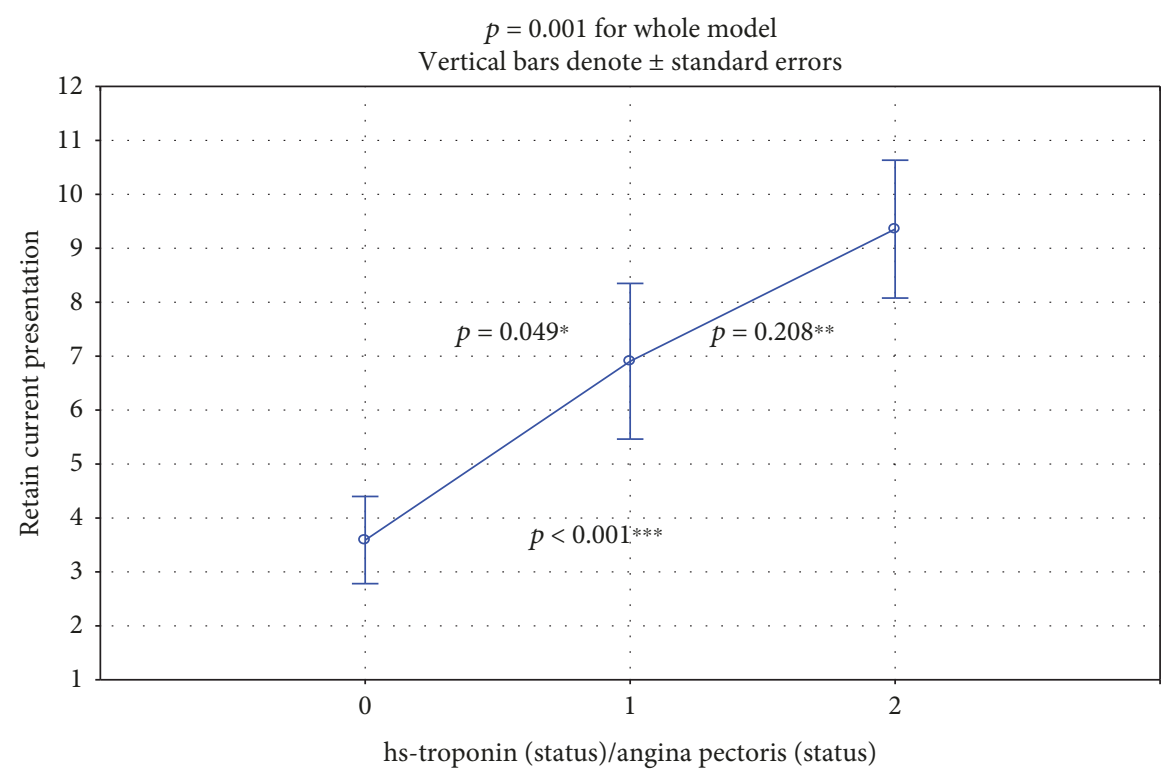

Figure 2: Comparison of NT-proBNP standardized according to age and sex between subgroup 0 (hsTnI-/AP-), subgroup 1 (hsTnI+/AP-), and subgroup 2 (hsTnI+/AP+). $p=0.001$ for the whole model. Inter-subgroup comparison: * subgroup 0 vs. $1: p=0.049 ;{ }^{* *}$ subgroup 1 vs. 2 : $p=0.208 ;{ }^{* * *}$ subgroup 0 vs. $2: p<0.001$.

The standardized value of NT-proBNP/ULN showed a gradual significant increase (hsTnI-/AP- vs. hsTnI+/AP- vs. hsTnI+/AP+: $3.59+0.63$ vs. $6.90+1.31$ vs. $9.35+1.87 ; p=$ 0.001 for the whole model in ANOVA; Figure 2).

In the comparison between subgroups (hsTnI-/AP- vs. hsTnI+/AP- vs. hsTnI+/AP+), two echocardiographic parameters increased significantly. The LV maximum wall thickness (LVMWT) at diastole was $1.99 \pm 0.08 \mathrm{~cm}$ vs. $2.28 \pm 0.13 \mathrm{~cm}$ vs. $2.49 \pm 0.15 \mathrm{~cm}$ (Figure 3, normal distribution, ANOVA test, $p=0.004$ in the whole-model analysis).

The maximal provoked LVOT gradient increased significantly in only the painful-ischemia subgroup: 11
(7-30) $\mathrm{mmHg}$ vs. $12(9.35-31.5) \mathrm{mmHg}$ vs. $100(43-120)$ $\mathrm{mmHg}$ (Figure 4; abnormal distribution: Kruskal-Wallis test, $p<0.001$ for the whole model).

The increase of LAD from subgroup to subgroup was nonstatistically significant $(4.28 \pm 0.16$ vs. $4.64 \pm 0.28$ vs. $4.95 \pm 0.15 ; p=0.051$ for the whole model, Figure 5).

NT-proBNP/ULN was more strongly correlated with echocardiographic parameters than NT-proBNP (Table 4).

In multiple linear regression analysis, resting LVOT gradient, LAD, and the presence of angina were identified as independent factors affecting NT-proBNP levels in patients with HCM (Table 5). 


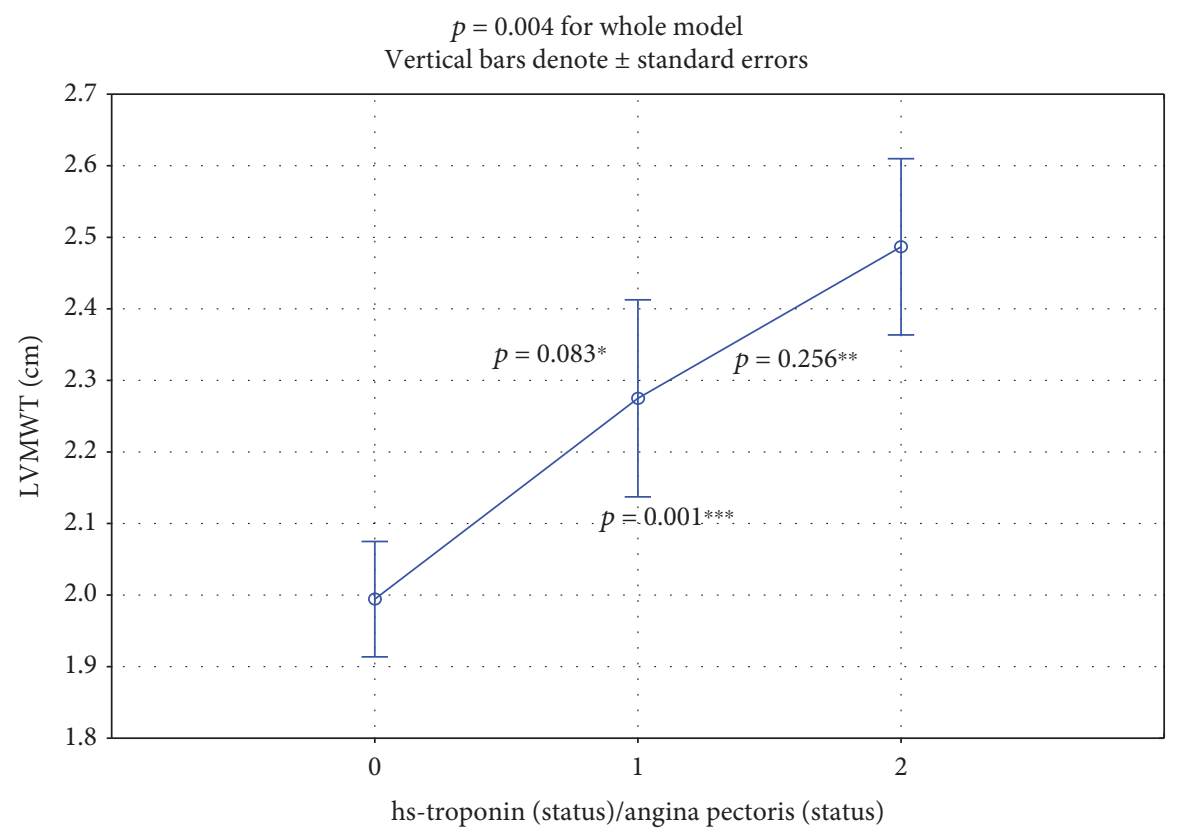

FIGURE 3: Significant increase $(p=0.004)$ of LVMWT in the whole model. Inter-subgroup comparison: ${ }^{*}$ subgroup 0 vs.1: $p=0.083$; ${ }^{* *}$ subgroup 1 vs. 2 : $p=0.256 ;{ }^{* * *}$ subgroup 0 vs. $2: p=0.001$.

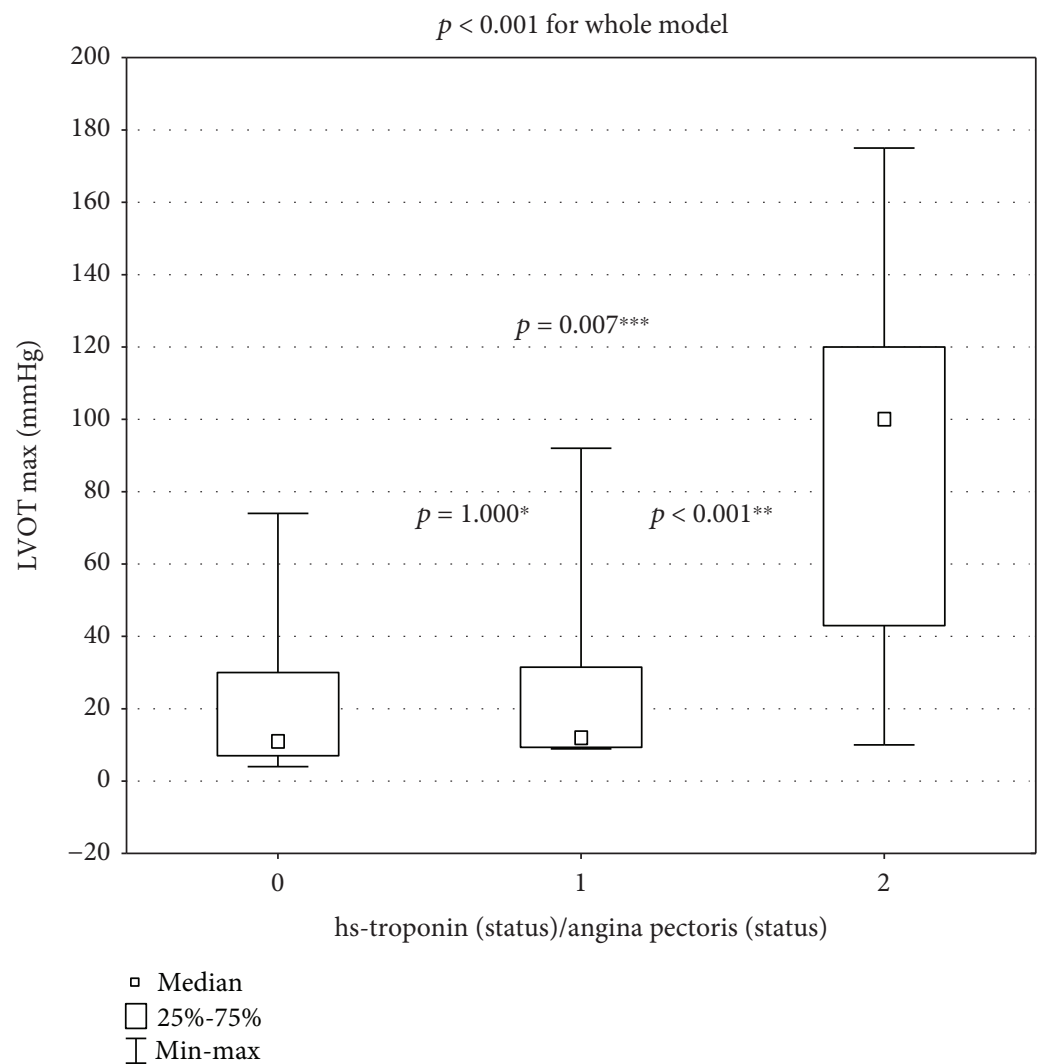

FIgURE 4: Significant increase ( $p<0.001$ for the whole model) of provocable LVOT gradient. Inter-subgroup comparison: * subgroup 0 vs. 1 : $p=1.000 ;{ }^{* *}$ subgroup 1 vs. $2: p<0.001{ }^{* * *}$ subgroup 0 vs. $2: p=0.007$. 


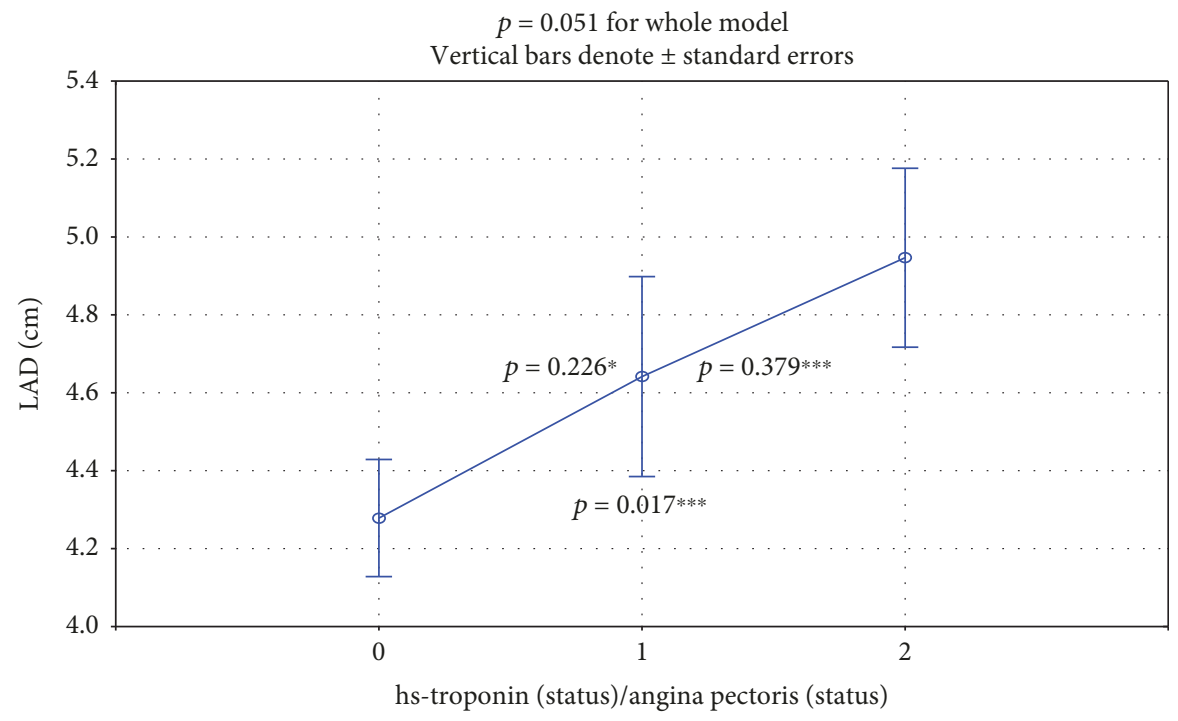

Figure 5: There was a nonsignificant increase in $\operatorname{LAD}\left(p=0.051\right.$ for the whole model). ${ }^{*}$ Subgroup 0 vs. $1: p=0.226 ;{ }^{* *}$ subgroup 1 vs. 2 : $p=0.379 ;{ }^{* * *}$ subgroup 0 vs. $2: p=0.017$.

TABLE 4: Correlations between NT-proBNP(/ULN) and echocardiographic parameters.

\begin{tabular}{lcc}
\hline & NT-proBNP & NT-proBNP/ULN \\
\hline LVMWT & $r=0.24^{*}$ & $r=0.36$ \\
LVOTG max & $r=0.44$ & $r=0.54$ \\
LAD & $r=0.41$ & $r=0.42$ \\
\hline
\end{tabular}

${ }^{*}$ Nonsignificant; remaining correlations were significant; $p<0.05$.

\section{Discussion}

hsTnI is a precise and very useful biomarker for the detection of even small, focal, subendocardial myocardial injury caused by ischemia in patients with HCM. In recent investigations, measurements of hsTnI levels were synchronized with a noninvasive assessment of clinical and hemodynamic parameters within a short time, similar with previous studies [7-11]. We have documented that levels of both NT-proBNP and NT-proBNP/ULN were the highest in the most ischemic subgroup (painful angina pectoris; hsTnI+/AP+). Both biomarkers and echocardiographic parameters have not been investigated previously using currently proposed models of analysis [12-15].

In the hsTnI+/AP+ subgroup, both LVMWT at diastole and the provocable LVOT gradient had significantly higher values. In the subgroup of painless ischemia, the LVMWT at diastole had an intermediate value and differed significantly from the values of the nonischemic and painful ischemic subgroups (Figure 3). Our findings are rational because both increased myocardial mass and LVOT gradient induce myocardial ischemia through an increase in oxygen demand. LAD is the third echocardiographic risk factor for sudden death included in the guideline calculator from the European Society of Cardiology $[4,10,11]$. This value was increased in subgroups, but the differences were only on the statistical borderline $(p=0.052$, Figure 5$)$.
In an experimental protocol for HCM with more physiological conditions [15], biomarkers were measured before exercise testing with only one control point at 4 hours postexercise. NT-proBNP increased by $27 \%$ after exercise. Similarly, hsTnI increased by $24 \% 4$ hours after exercise, but the differences were not statistically significant. The 4-hour check-point in the postexercise recovery seems to be too long for peak NT-proBNP and too short for peak hsTnI values. In small study with 7 young HCM patients without symptomatic coronary artery disease, authors [16] detected elevated troponin levels after physical exercise in 5 patients. In serial measurement, the peak concentration had been reached between 6 and 9 hours and levels returned to preexercise values within 24 hours. Troponin release was consistently diminished after use of a beta-blocker. We tried to study this problem by using a 24-hour spectrum of time and with a more physiological approach. We monitored the 24-hour physical activity and occurrence of angina. It has been proposed that moderate, fluctuating exercise during daily physical activity may be more appropriate to detect abnormalities in cardiac biomarker release, rather than maximum symptom-limited exercise [15]. To support the theory about the link with postexercise prolonged myocardial ischemia, we need a larger study, with many points in time to measure hsTnI (24-hour profile of release). Tesic et al. [17] recently found that the coronary flow reserve in the left anterior descending artery appeared to be an independent predictor of NT-proBNP. Thus, elevated NT-proBNP might be the result of cardiac ischemia indicated by low coronary flow reserve.

4.1. Limitations. The main limitation of study is the relatively small number of patients due to several exclusion criteria. Not all of the patients underwent coronary arteriograms, which was only performed on patients with the appropriate indications. The minority of patients did not have coronary angiography performed (young, without $\mathrm{AP}$, and without 
TABLE 5: Results of linear regression analysis.

\begin{tabular}{lcccc}
\hline Independent variable & Coefficient & Standard error & Standardized coefficient & $p$ value \\
\hline Resting LVOT gradient & 19.76 & 5.29 & 0.41 & $<0.001$ \\
LAD & 314.44 & 123.30 & 0.26 & 0.013 \\
Angina & 658.35 & 297.13 & 0.25 & 0.031 \\
\hline
\end{tabular}

risk factors for $\mathrm{CAD}$ ). The risk for $\mathrm{CAD}$ was minimal and coronary angiography was not indicated. The strategy of noninvasive identification of subgroups with low likelihood of obstructive CAD [18] is effective.

The next limitation of the study was the following problem. We measured biomarkers simultaneously, once in time, and we do not have a sufficient period of follow-up with prognostic findings.

\section{Conclusions}

Both painless ischemia and painful ischemia are associated with a gradual, significant increase in NT-proBNP/ULN in comparison to the double-negative hsTnI/AP subgroup. In contrast, NT-proBNP was significantly higher in only the subgroup with painful ischemia. In the comparison between subgroups (hsTnI-/AP- vs. hsTnI+/AP- vs. hsTnI+/AP+), two echocardiographic parameters increased significantly: LVMWT at diastole (in the whole-model analysis) and the maximal provoked LVO T gradient (only in the painful subgroup in both analysis models).

\section{Data Availability}

The data used to support the findings of this study are available from the corresponding author upon request.

\section{Additional Points}

Clinical Perspective. The study documented the evidence that positive troponin status is timely related with higher biomarker value NT-proBNP reflecting LV myocardial dysfunction. From the clinical perspective, monitoring of these 2 biomarkers may improve pharmacological and nonpharmacological treatments and safety of exercise training.

\section{Conflicts of Interest}

The authors declare that they have no conflicts of interest.

\section{Authors' Contributions}

RR-S performed the conceptualization, formal analysis, investigation, writing of the reviews, and editing. AG performed formal analysis, investigation, writing of the reviews, and editing. $\mathrm{AD}$ performed some analysis, investigation, and editing, and PPD performed the conceptualization, formal analysis, investigation, draft writing, writing of the reviews, and editing. RR-S and AG contributed equally to this work.

\section{References}

[1] D. W. Kehl, A. Buttan, R. J. Siegel, and F. Rader, "Clinical utility of natriuretic peptides and troponins in hypertrophic cardiomyopathy," International Journal of Cardiology, vol. 218, no. 2, pp. 252-258, 2016.

[2] M. Gawor, M. Śpiewak, J. Janas et al., “The usefulness of sST2 and galectin-3 as novel biomarkers for better risk stratification in hypertrophic cardiomyopathy," Kardiologia Polska, vol. 75, no. 10, pp. 997-1004, 2017.

[3] T. Kubo, H. Kitaoka, M. Okawa et al., "Combined measurements of cardiac troponin I and brain natriuretic peptide are useful for predicting adverse outcomes in hypertrophic cardiomyopathy," Circulation Journal, vol. 75, no. 4, pp. 919-926, 2011.

[4] Authors/Task Force members, P. M. Elliott, A. Anastasakis et al., "2014 ESC guidelines on diagnosis and management of hypertrophic cardiomyopathy: The task force for the diagnosis and management of hypertrophic cardiomyopathy of the European Society of Cardiology (ESC)," European Heart Journal, vol. 35, no. 39, pp. 2733-2779, 2014.

[5] A. Pelliccia, E. Lemme, V. Maestrini et al., "Does sport participation worsen the clinical course of hypertrophic cardiomyopathy? Clinical outcome of hypertrophic cardiomyopathy in athletes," Circulation, vol. 137, no. 5, pp. 531-533, 2018.

[6] J. L. Blackshear, R. E. Safford, C. S. Thomas et al., "Platelet function analyzer 100 and brain natriuretic peptide as biomarkers in obstructive hypertrophic cardiomyopathy," The American Journal of Cardiology, vol. 121, no. 6, pp. 768774, 2018.

[7] A. Gębka, R. Rajtar-Salwa, A. Dziewierz, and P. Petkow-Dimitrow, "Painful and painless myocardial ischemia detected by elevated level of high-sensitive troponin in patients with hypertrophic cardiomyopathy," Advances in Interventional Cardiology, vol. 14, no. 2, pp. 195-198, 2018.

[8] R. Rajtar-Salwa, R. Hładij, and P. P. Dimitrow, "Elevated level of troponin but not N-terminal probrain natriuretic peptide is associated with increased risk of sudden cardiac death in hypertrophic cardiomyopathy calculated according to the ESC Guidelines 2014," Disease Markers, vol. 2017, Article ID 9417908, 5 pages, 2017.

[9] R. Hładij, R. Rajtar-Salwa, and P. Petkow Dimitrow, “Associaton of elevated troponin levels with increased heart rate and higher frequency of nonsustained ventricular tachycardia in hypertrophic cardiomyopathy," Polish Archives of Internal Medicine, vol. 126, no. 6, pp. 445-447, 2017.

[10] R. Hładij, R. Rajtar-Salwa, and P. P. Dimitrow, "Troponin as ischemic biomarker is related with all three echocardiographic risk factors for sudden death in hypertrophic cardiomyopathy (ESC Guidelines 2014)," Cardiovascular Ultrasound, vol. 15, no. 1, p. 24, 2017.

[11] C. M. McGorrian, S. Lyster, A. Roy et al., "Use of a highlysensitive cardiac troponin I assay in a screening population 
for hypertrophic cardiomyopathy: a case-referent study," BMC Cardiovascular Disorders, vol. 13, no. 1, p. 70, 2013.

[12] S. W. Kim, S. W. Park, S. H. Lim et al., "Amount of left ventricular hypertrophy determines the plasma $\mathrm{N}$-terminal pro-brain natriuretic peptide level in patients with hypertrophic cardiomyopathy and normal left ventricular ejection fraction," Clinical Cardiology, vol. 29, no. 4, pp. 155-160, 2006.

[13] G. Kahveci, F. Bayrak, B. Mutlu, and Y. Basaran, "Determinants of elevated NT-proBNP levels in patients with hypertrophic cardiomyopathy: an echocardiographic study," Heart, Lung and Circulation, vol. 18, no. 4, pp. 266-270, 2009.

[14] Y. Sato, R. Taniguchi, K. Nagai et al., "Measurements of cardiac troponin $\mathrm{T}$ in patients with hypertrophic cardiomyopathy," Heart, vol. 89, no. 6, pp. 659-660, 2003.

[15] J. E. Ho, L. Shi, S. M. Day et al., "Biomarkers of cardiovascular stress and fibrosis in preclinical hypertrophic cardiomyopathy," Open Heart, vol. 4, no. 2, article e000615, 2017.

[16] G. A. Pop, E. Cramer, J. Timmermans, H. Bos, and F. W. Verheugt, "Troponin I release at rest and after exercise in patients with hypertrophic cardiomyopathy and the effect of betablockade," Archivos de Cardiologia de Mexico, vol. 76, no. 4, pp. 415-418, 2006.

[17] M. Tesic, J. Seferovic, D. Trifunovic et al., "N-terminal probrain natriuretic peptide is related with coronary flow velocity reserve and diastolic dysfunction in patients with asymmetric hypertrophic cardiomyopathy," Journal of Cardiology, vol. 70, no. 4, pp. 323-328, 2017.

[18] J. Reeh, C. B. Therming, M. Heitmann et al., "Prediction of obstructive coronary artery disease and prognosis in patients with suspected stable angina," European Heart Journal, vol. 40, no. 18, pp. 1426-1435, 2019. 


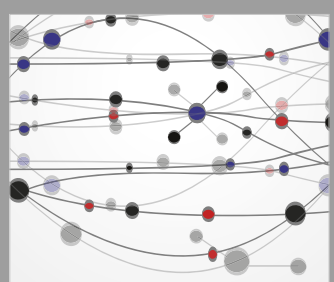

The Scientific World Journal
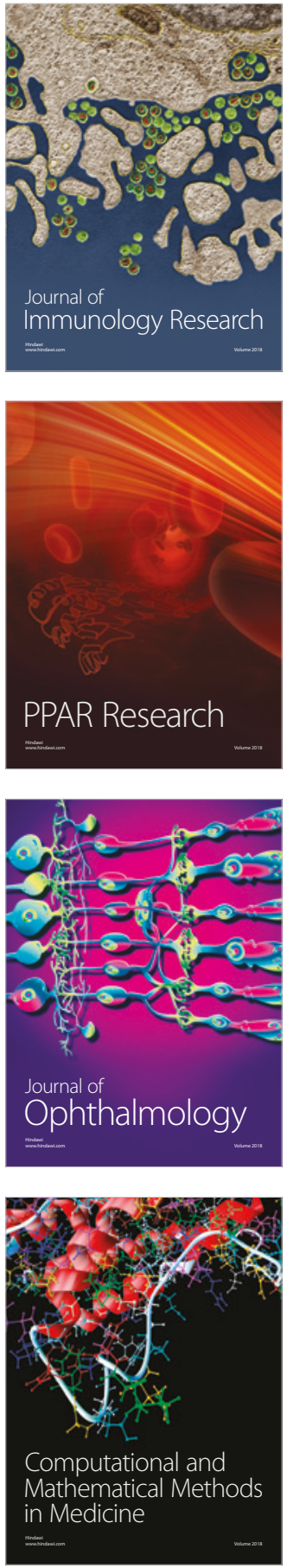

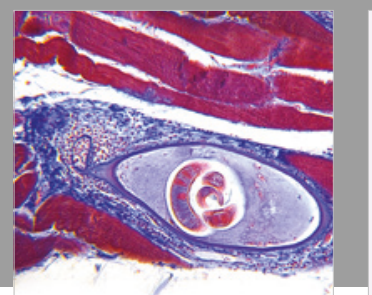

Gastroenterology Research and Practice

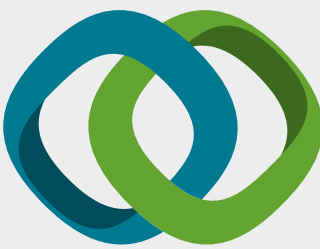

\section{Hindawi}

Submit your manuscripts at

www.hindawi.com
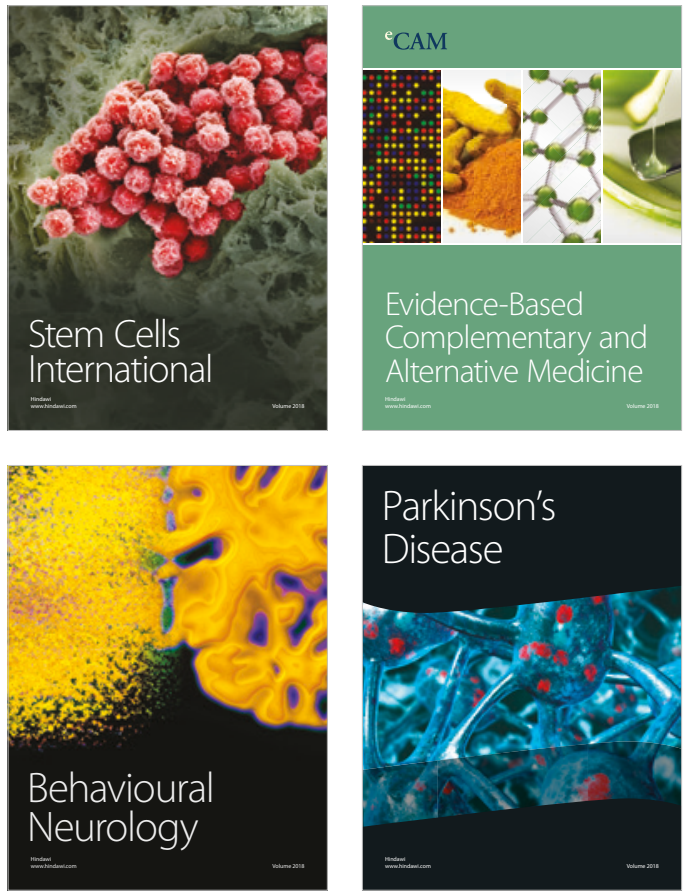

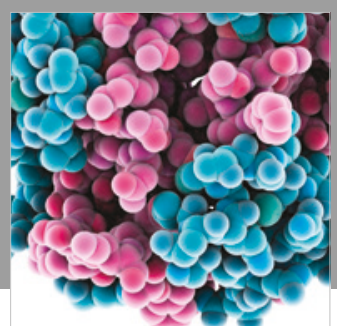

ournal of

Diabetes Research

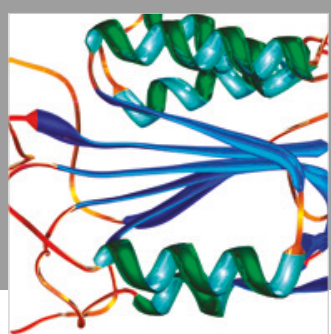

Disease Markers
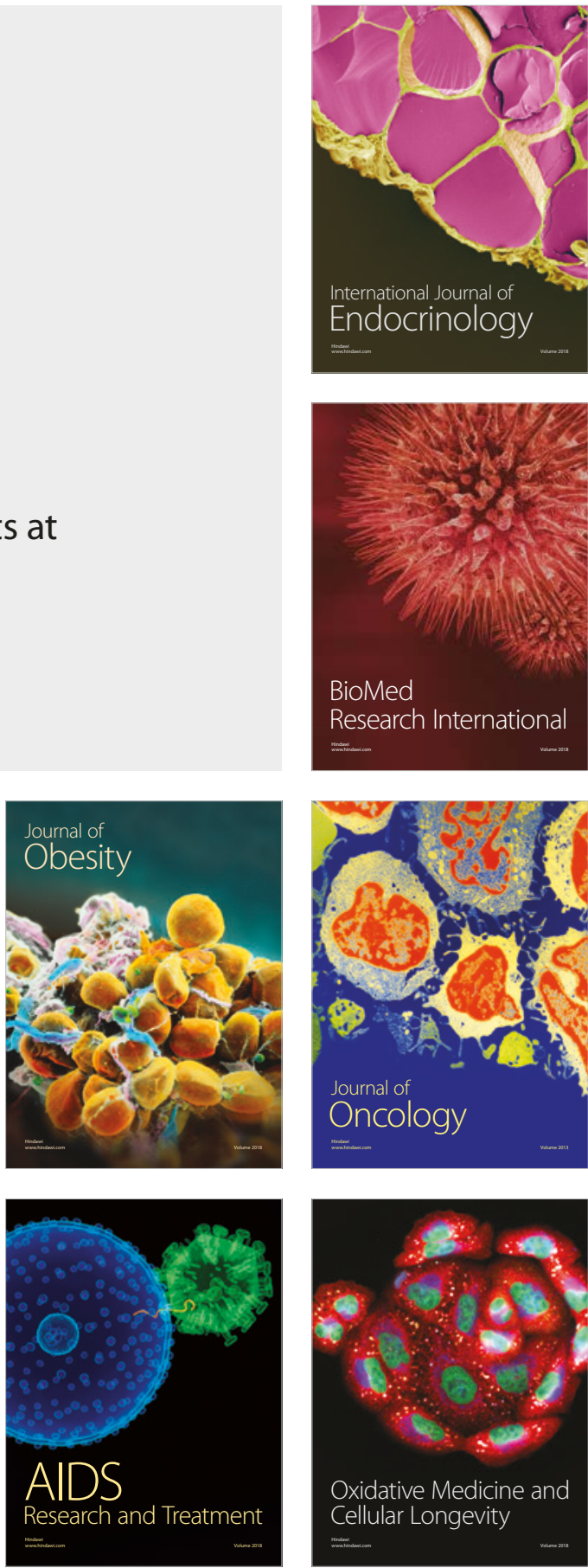